\title{
Development of a Compact Hybrid Underwater Vehicle Using Variable Vector Propeller
}

\author{
Yutaka Nagashima \\ Dept. of Electrical Electronic Eng. \\ Sasebo National College of Tech. \\ 1-1 Okishin-machi Sasebo, Nagasaki \\ nagasima@sasebo.ac.jp \\ Takakazu Ishimatsu \\ Dept. of Mechanical Systems Eng. \\ Nagasaki University \\ 1-14 Bunkyo-machi, Nagasaki \\ ishi@net.nagasaki-u.ac.jp
}

\author{
Nobuyoshi Taguchi \\ Dept. of Mechanical Eng. \\ Industrial Technology Center of Nagasaki \\ 2-1303-8 Ikeda Ohmura, Nagasaki \\ taguchi@tc-nagasaki.pr.jp
}

\begin{abstract}
:
This paper describes an autonomous underwater vehicle (AUV) with fuzzy controller and PID controller. Our AUV is compact and light in weight by employing variable vector (VARIVEC) propeller and compact controller. The AUV is autonomously controlled by utilizing the electronic compass, collision avoidance sonar, depth sensor and GPS receiver unit. A fuzzy controller and PID controller have designed and examined in autonomous navigation. Experimental results indicate that our AUV can move along the targeted path and hold the desired position and the depth. Furthermore, we have responded to demand of fisheries and environmental survey companies, the untethered remotely operated vehicle (UROV) is developed for monitoring the real-time image under the sea. The feature of the UROV is controlled utilizing both of wireless and optical fiber. The UROV is controlled smoothly and clearly image is captured by remote control method. We can survey the buried cultural property in the field of maritime archaeology.
\end{abstract}

Keywords: AUV, UROV, Variable vector propeller, Maritime archaeology

\section{INTRODUCTION}

The scientific investigations of marine environment often require the use of underwater vehicles to perform survey, monitoring, and data collection tasks. We are primarily concerned with daily marine measurements in the closed sea areas like the Isahaya Bay and the Ohmura Bay in Nagasaki prefecture, Japan. Usually underwater vehicle are equipped with many thrusters. The thruster control mechanism is complicated and this takes a larger weight ratio, making the AUV larger and heavier (URA, 1997). We developed the compact VARIVEC propeller (Hoshino, 1991) system utilizing the R/C helicopter elements, which are swash plate and DC servos. The VARIVEC propeller can generate six components including thrust, lateral force and moment by changing periodically the blade angel of the propeller during one revolution. It is possible to reduce the number of thrusters, mechanism and hence power sources. We developed a compact and light AUV by employing VARIVEC propeller and a compact control system using complex programmable logic device (CPLD). A compact AUV is capable of performing several challenging functions and enables it to be used by a limited number of persons. For the daily measuring tasks, a small AUV will be necessary for efficient exploration and investigation of a wide range of a sea. We have implemented three modules, which include the receiver control module, the sensor control module and the propeller control module. Each module utilizes one-chip microcomputer as the distributed control system for the AUV. Depending upon the width of pulse width modulation (PWM) pulse, servos rotate in clockwise or counter clockwise direction, controlling the motion of the blades of VARIVEC propeller. We have used the electronic compass, collision avoidance sonar, depth sensor and GPS receiver for autonomous navigation. The electronic compass includes compass, two-axis tilt sensor, three-axis magnetometer and temperature sensor.

Furthermore, we have responded to the demands of fisheries and marine environmental survey companies. We tried to design and manufacture the UROV using VARIVEC propeller. The control system is configured utilizing the combination of the optical fiber and the wireless system. The wireless system is in the buoy that includes the $\mathrm{R} / \mathrm{C}$ receiver, image transmitter and wireless data modem. The optical fiber is connected the UROV propeller driver and image transmitter via EO (electrical-optical) or OE (optical-electrical) converter. The moving image is captured by the video movie or digital cameras. The many images are remotely exchanged by the controller. The UROV is controlled by the R/C transmitter simply.

To evaluate the compact AUV, we have carried out the autonomous navigation tests at the Sasebo National College of Technology in an anechoic water tank, which suppresses the reflection effects of the wall surface. Also, 
we tried the test in the deep-sea tank of Kyushu University Research Institute for Applied Mechanics and a sea. Experimental results indicated the effectiveness of our approach and the AUV could move along the targeted path and hold the desired position.

To evaluate the UROV, we have tried to survey the buried cultural property in maritime archaeology at the GOTO islands in Nagasaki prefecture and investigate the canal of the HUIS TEN BOSCH resorts. The mission is carried out smoothly and the underwater image is captured remote control system. In this paper, we reported the experimental results and discussions about it.

\section{SYSTEM CONFIGURATION}

\subsection{AUV system}

Developed AUV is shown in Fig.1. The front of AUV is mounted by acrylic dome in order to observe the undersea. The overall length is $85 \mathrm{~cm}$ and the gross weight is $75 \mathrm{Kgs}$. The AUV is equipped a VARIVEC propeller in the rear side. In the control system, we have implemented three modules, which include:

(1) Receiver control module: This module controls the reception of the mission command from the support vessel and transmits the signal as transponder for positioning system.

(2) Sensor control module: This module controls the various data acquisition techniques and recognized the surroundings of the AUV for autonomous navigation.

(3) VARIVEC propeller control module: This module controls the final decision of mission command and generates the control data for VARIVEC propeller system by using the CPLD circuit. The control algorithm is designed by the fuzzy control and PID control method in order to increase the intelligence and the smooth navigation.

Each module utilizes one-chip microcomputer as the distributed control system for the vehicle. The mission command is generated by the microcomputer on the support vessel and it generates the necessary control data for the CPLD as sophisticated logic circuit, which in turns produces the pulse width modulation signal to rotate the DC servos and hence controls the VARIVEC propellers. Table1 shows the dimensions of the AUV and the principal particulars of the VARIVEC propeller. The system configuration of the AUV is shown in Fig.2. This control system increases the serviceability, flexibility and reliability by the use of the distributed control method. They provide a more robust operation in real-time than the traditional approach which includes a sequence of environmental analysis, planning and execution.
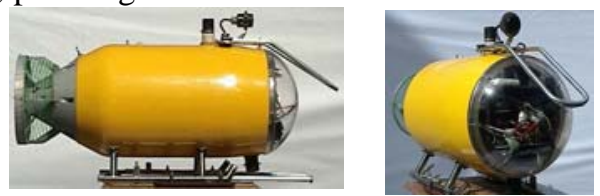

Fig.1 Compact AUV
Table 1 Dimensions and principal particulars of AUV

\begin{tabular}{|l|l|l|l|}
\hline \multicolumn{2}{|c|}{ AUV } & \multicolumn{2}{c|}{ VARIVEC propeller } \\
\hline Length & $865 \mathrm{~mm}$ & Diameter & $210 \mathrm{~mm}$ \\
\hline Width & $350 \mathrm{~mm}$ & Expanded Area Ratio & 0.293 \\
\hline Height & $520 \mathrm{~mm}$ & Boss Ratio & 0.476 \\
\hline Weight & $75 \mathrm{~kg}$ & Blade Number & 4 \\
\hline Depth & $20 \mathrm{~m}$ & Rake Angle & 0 degree \\
\hline Seed & $0.8 \mathrm{knots}$ & Number of revolution & $370 \mathrm{rpm}$ \\
\hline
\end{tabular}

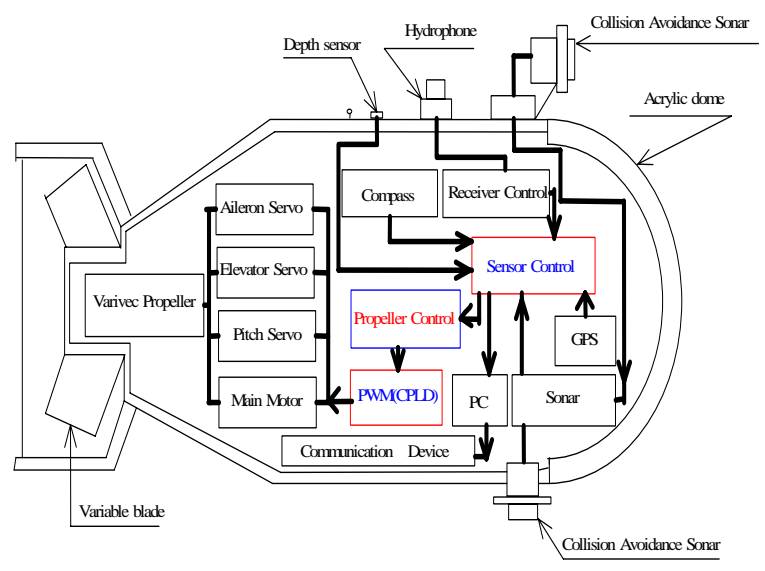

Fig.2 Block diagram of AUV system configuration

We have used the electronic compass, the depth sensor, the collision avoidance sonar and GPS receiver unit for autonomous navigation. The electronic compass includes compass, two-axis tilt sensor, three-axis magnetometer and temperature sensor. The electronic compass is used to measure the bearing, roll angles and pitch angles during navigation. The collision avoidance sonar incorporates four channels for the front, both sides and the bottom using 150 $\mathrm{kHz}$ hydrophones. It is used to measure the distance from the obstacle and from the sea floor. The resolving power of the sonar is about $5 \mathrm{~cm}$. We used the pressure sensor in order to measure the depth. The accuracy of the depth sensor is under $0.01 \%$ and the range is within $50 \mathrm{~m}$. The GPS receiver unit is used to measure the absolute position as the latitude and the longitude during the staying of the AUV on the sea surface.

It is important that the AUV has the highly reliable acoustic communication between the support vessel and the AUV. We have achieved the communication modules by using the newly designed amplifiers and filters. The module can use two modes of transponder, i.e. for positioning system and for mission command communication using $50 \mathrm{kHz}$ hydrophone. At first the module measures the range and the bearing between the AUV and the support vessel, and then the mission command is sent to the AUV. 


\subsection{UROV system}

In order to observe real-time image in the underwater, we have developed an UROV system that utilizing the combination of optical fiber and wireless system. The UROV system, video camera housing and new propeller blade are shown in Fig.3. The control command of the UROV is transmitted using PCM code by the R/C transmitter. The control command is communicated via the optical fiber to the four servos configuring VARIVEC propeller. The moving picture is captured by the video camera and remotely photographing is controlled by the $\mathrm{R} / \mathrm{C}$ transmitter switch. The sensor data of the UROV are communicated via wireless modem in the buoy. The optical fiber is winded and released by automatically or wireless remote control.

The difference of AUV and UROV is the blade shape. The UROV blade is heptagon which is designed in order to save the power source and increase the propeller efficiency.
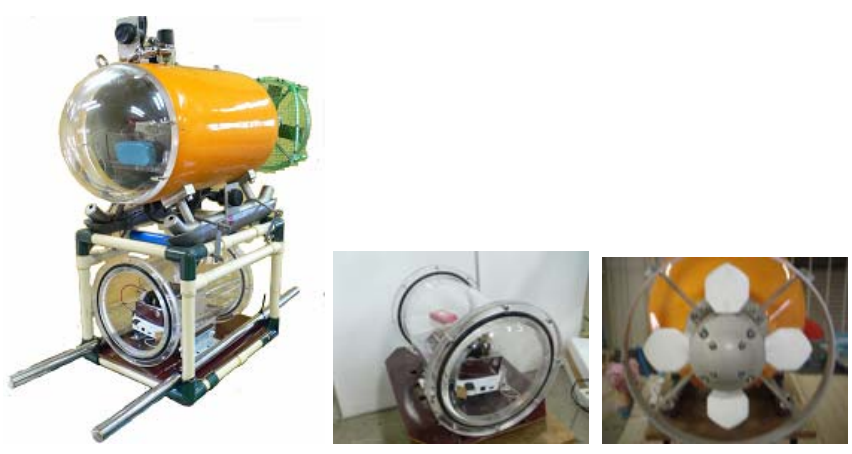

Fig.3 UROV system, camera housing and propeller blade

The blade of AUV is the NACA series and the conventional rectangular shape with end plate increasing the thrust.

The block diagram of the communication system is shown in Fig.4. The UROV motion controller is PCM modulation transmitter of R/C helicopter system. The transmitter has nine channels. The VARIVEC propeller use four channels as aileron servo, elevator servo, pitch servo, and main motor. The other channels are remote photographing, remote zoom, ballast releaser and the exchanging mode of AUV or UROV.

In the buoy, the $\mathrm{R} / \mathrm{C}$ receiver is connected to the electrical -optical (EO) converter which transforms an electrical signal into an optical signal for communication using optical fiber. The fiber cable connects the camera housing attached under the UROV. The optical-electrical (OE) converter transforms an optical signal into an electrical signal in the camera housing. There are two types of $\mathrm{OE}$ and EO converters in order to communicate the control signal and image signal. We used the time division multiplexer (TDM) type converter for sensor data and image data communication.

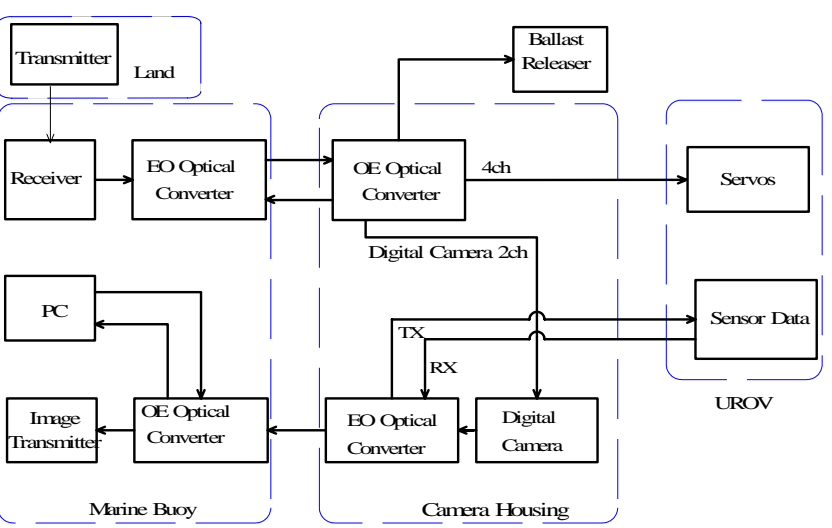

Fig.4 Block diagram of UROV communication system

\section{VARIABLE VECTOR PROPULSION SYSTEM}

Usually, underwater vehicle are equipped with many thrusters to move forward or backward, upward or downward, turning or a combination of them, for three-dimensional maneuvering. In our research, we have employed VARIVEC propeller as a propulsion system for the underwater vehicle. It thus becomes possible to reduce the number of propellers by VARIVEC propeller without losing any maneuverability. This has also made it possible to the construction of compact and light AUV. The advantage of the using of the VARIVEC propeller lies in their capability to generate thrust and moment continuously in any of the three-dimensional directions by controlling the collective pitch, cyclic pith and combination of the both. Moreover, they have a fast response to the slightest position change of the underwater vehicle. Mechanical realization of VARIVEC propeller system is very difficult to achieve. To solve this problem, we have used the mechanical assembly links and servos elements generally used in R/C helicopters. This approach has enabled us to build a very compact and effective mechanical system, which is distinctly different from the existing usable techniques. In our system, VARIVEC propeller is assembled to swash plate, which is linked to aileron motion, elevator motion and pitch motion control servos. There is another servomotor for the control of main drive motor for propeller shaft. The basic motion of VARIVEC propeller is as follows:

(1) Neutral pitch: The swash plate is in neutral position, the blades set are neutral too and propeller doesn't generate thrust.

(2) Collective pitch: All blades set or changed to the same pitch angle simultaneously. The propeller generates the forwards or backward thrust.

(3) Cyclic pitch: Each blade changes pitch during each cycle or rotation of the hub. The propeller generates the upward, downward, left and right thrust.

(4) Combination pitch: Collective pitch and cyclic pitch is achievable. The function of the propeller is to provide a thrust vector, as required, using both the cyclic and the collective pitch. The AUV can move any direction smoothly. 
The assembly of the VARIVEC propeller is shown in Fig.5. The servos for the CPLD control the VARIVEC propeller motions. Servos for their operation need a trigger pulse at the intervals of about 14.2 milliseconds. The CPLD can generate the PWM pulses simultaneously for all servos. Depending upon the width of the PWM pulse, servos rotate in clockwise or counter clockwise direction, controlling the movement of the blades of the VARIVEC propellers. Main drive servomotor also operates on the same principle. If the pulse width is wide, it rotates slowly and if it is narrow, it speeds up.
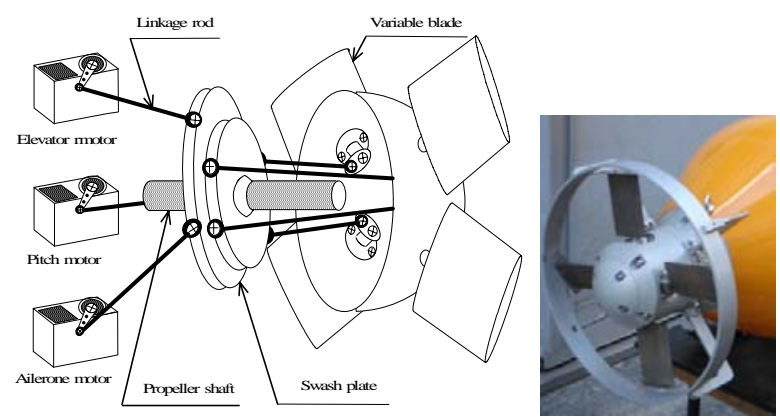

Fig.5 Assembly of VARIVEC propeller

\section{CONTROL METHODS}

\subsection{Fuzzy controller}

When we tried the feedback control method on the autonomous navigation, the trajectory was zigzag and expended the time. In order to improve the control ability of autonomous navigation, we implemented the fuzzy logic control in the distributed controller. The benefit of fuzzy logic approach for the AUV is better matching of the control strategy. The modification of the controller is not required a dynamic model, thus allowing for rapid development of a working design and less sensitivity to plant variations. The fuzzy logic rules specification for the AUV is defined in the Table2. We designed 147 rules for the bearing, the depth and the collision avoidance. In order to increase the accuracy, the antecedent membership function is double-checked using the error from the target path and the rate of change. The antecedent membership functions are designed to 7 type's triangles. The algorithm is correspondingly designed to the errors to target bearing and the rate of changed bearing. Both membership functions have various gradients in order to obtain the most suitable condition. The input and the output rules are included bearing, depth and sonar conditions. The conclusion membership function is singleton. The inference method is used by a Min-Max method. The defuzzification is used by a simplified center of gravity method.

The block diagram of fuzzy controller is shown in Fig.6.
Table 2 Fuzzy rules specification

\begin{tabular}{|l|l|}
\hline Rules & 147 \\
\hline Number of input/output & Input: 9, Output: 4 \\
\hline Antecedent membership function & Triangle \\
\hline Conclusion membership function & Singleton \\
\hline Inference method & Min-Max method \\
\hline Defuzzification & Center of gravity method \\
\hline
\end{tabular}

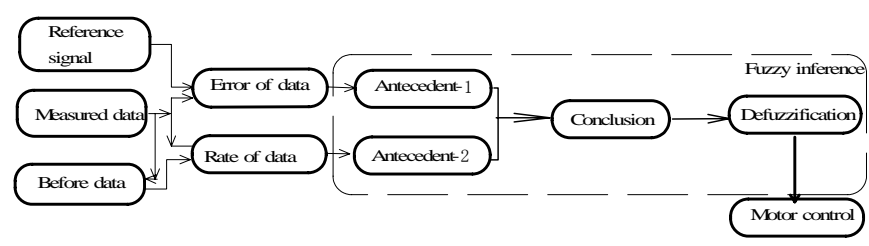

Fig.6 Block diagram of fuzzy controller

\subsection{PID controller}

In order to realize stable autonomous navigation, we tried to implement the PID algorithm as shown in Fig.7. All parameters including the proportional (P), integral (I), and derivative (D) are decided by the simulations of AUV, the final parameters are fine-tuned by way of experiment. The algorithm is adapted by the potential type as follows:

$$
M=\frac{100}{P B}\left(e_{n}+\frac{1}{T_{I}} \sum_{i=0}^{n} e_{i} \Delta T+T_{D} \frac{e_{n}-e_{n-1}}{\Delta T}\right)
$$

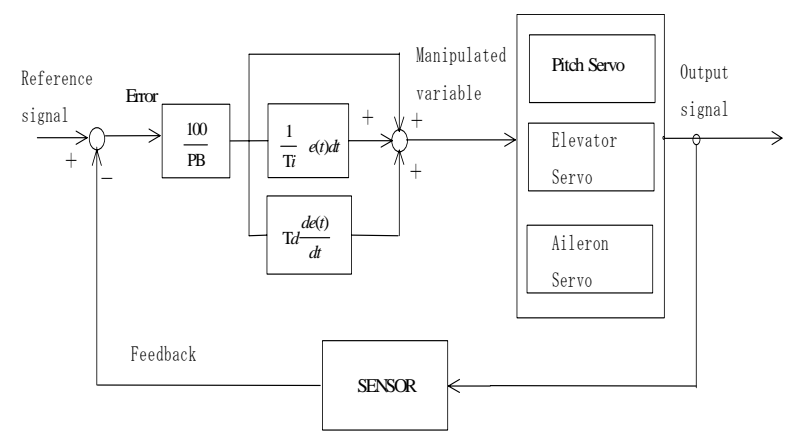

Fig.7 Block diagram of PID controller

\section{EXPERIMENTAL RESULTS AND DISCUSSIONS}

\subsection{AUV mode}

To evaluate the developed AUV system, we have conducted tests in an anechoic water tank (length $=10 \mathrm{~m}$, width $=5 \mathrm{~m}$, depth=4.5m), which has suppressed wall reflection. The anechoic water tank is situated at the Sasebo National College of Technology. The motion of AUV is confirmed with eyes. We have also recorded on the personal computer the bearing, two-axis tilt, three-axis magnetometer data and depth in order to evaluate the vehicle motion characteristics. 
(1) Feedback controller

The first mission of autonomous experiment is moving back and forth between two points that are apart from each other by a distance $10 \mathrm{~m}$ in the anechoic water tank and moving around turning in steps of 90 degrees in a $25 \mathrm{~m}$ swimming pool. The experimental results of autonomous navigation using feedback control on bearing are shown in the Fig.8. The AUV is moved back and forth following to the zigzag and unstable trajectory.

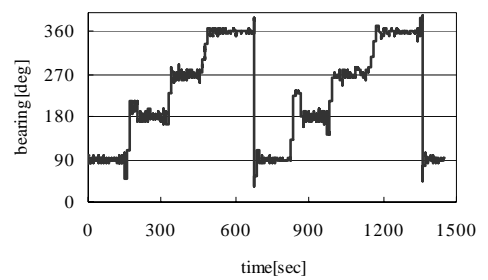

Fig.8 Results of feedback controller

\section{(2) Fuzzy controller}

The autonomous navigation results of same missions under fuzzy controller are shown in Fig.9. The examination of fuzzy control is carried out in the deep-sea tank (length $=65 \mathrm{~m}$, width $=5 \mathrm{~m}$, depth $=7 \mathrm{~m}$ ) of Kyushu University Research Institute for Applied Mechanics.

The experimental results are further improved than feedback algorithm experiments. Each result is indicated the good trajectory along the target bearing and depth.

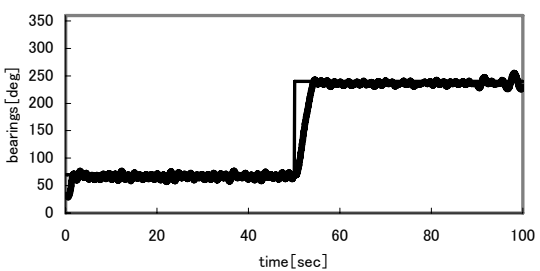

(a) Still water

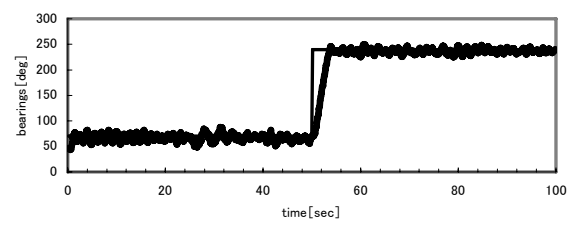

(b) Wave height $3(\mathrm{~cm})$

Fig.9 Result of fuzzy controller (bearing)

The Fig.10 shows the pitching angle and rolling angle of the AUV by measured inclinometer. In this case the wave height is $3(\mathrm{~cm})$ in the deep-sea tank. The depth of autonomous navigation is shown in Fig.11.

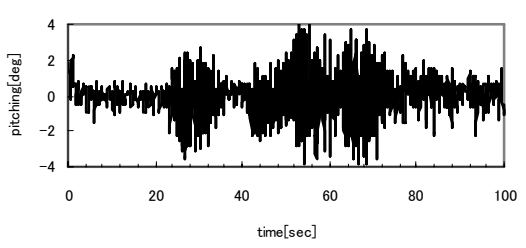

(a) Pitching angle

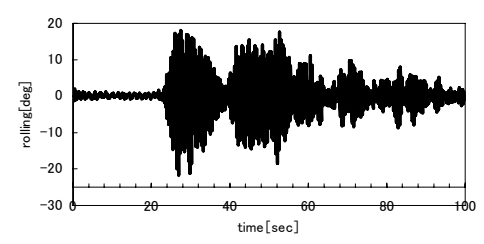

(b) Rolling angle

Fig.10 Results inclinometer through wave $(3 \mathrm{~cm})$

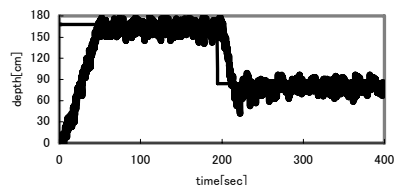

Fig.11 Results of fuzzy controller (depth)

(3) PID controller

The designed PID controller is examined to evaluate the autonomous navigation in case of the target bearing and the depth. We have carried out about autonomous navigation of P (proportional) control and PI (Proportional and Integral) control for fine-tuning of the parameters. The experimental results are shown in Fig.12 - Fig.13. In this case, the parameters of bearing are as follows:

$\mathrm{Kp}=0.001, \mathrm{Ti}=100, \mathrm{Td}=175$

The parameters of depth are as follows.

$$
\mathrm{Kp}=0.001, \mathrm{Ti}=0.001, \mathrm{Td}=40
$$

Kp means proportional gain, $\mathrm{Ti}$ and $\mathrm{Td}$ means integral and derivative time respectively. The results are further improved than feedback control on autonomous navigation.

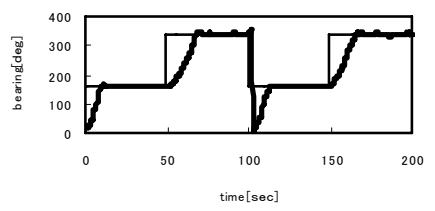

(a) Bearing (tank)

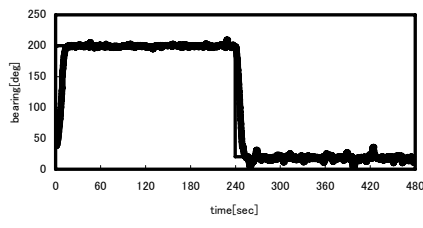

(b) Bearing (sea)

Fig.12 Results of PID controller 


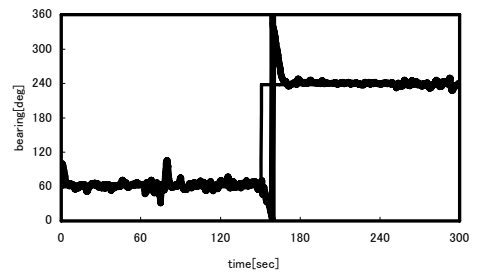

(c) Bearing (sea)

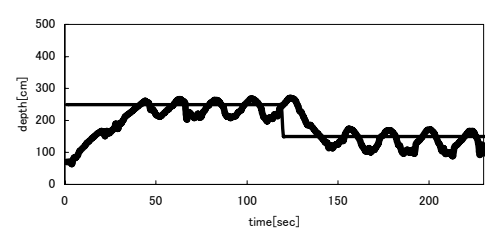

(d) Depth (sea)

Fig.13 Results of integration of two sensors

\subsection{UROV Mode}

We have controlled the UROV using R/C transmitter. The operator controls R/C transmitter lever switch or button for UROV motion and remotely photographing.

We have succeeded in making many performance experiments. The sensor data of the UROV is sent to the operator via optical fiber and wireless system. The compass and GPS data are shown in Fig.14.

We have tried to survey the buried cultural property in a maritime archaeology. One of a scene is shown in Fig. 15.

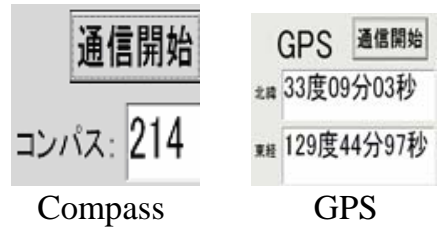

Fig.14. Remote monitoring results of sensors
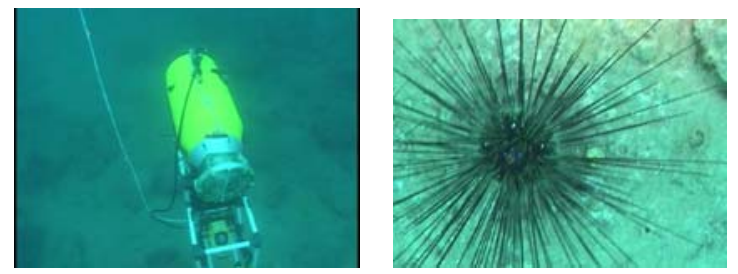

Fig. 15 Photographs of maritime archaeology survey

\section{CONCLUSIONS}

We developed a compact and light autonomous underwater vehicle by employing VARIVEC propeller and a compact control system. The experimental results indicated the effectiveness of our approach. The control of AUV was realized using precious fuzzy algorithm and PID algorithm. The AUV is in the improvement stage for practical application at the sea.
Furthermore, we have responded to demand of fisheries and environmental companies the UROV is developed for monitoring the real-time image under the sea. We could survey the buried cultural property in the field of maritime archaeology.

Future works are as follows:

(1) The navigation mode could be controlled semi-automatically.

(2) The performance of graphical user interface for the vehicle operator could be improved.

(3) The weight of vehicle could be decreased for one man operation, and so on.

\section{ACKNOWLEDGEMENTS}

The research work presented in this paper has been supported by the Kyushu University Research Institute of Applied Mechanics. Especially, the first author grateful to professor W.Koterayama and assistant professor M.Nakamura.

\section{REFERENCES}

[1] T.Hoshino: VARIVEC (Variable Vector) Propeller $4^{\text {th }}$. Propulsion Engineering Symposium, pp 391 - 405. (1991).

[2] Y.Nagashima, Jamal, T.M. and T.Ishimatsu: Implementation of a Distributed Control System for Autonomous Underwater Vehicle with VARIVEC Propeller, Proc. of KACC’99, pp E9 - E12. (1999).

[3] Y.Nagashima, Jamal, T.M., T.Ishimatsu and T.Mizokami: A Fundamental Control of Untethered Underwater Vehicle Using Variable Vector Propeller, $1^{\text {st }}$. Report Implementation of Vehicle System and Tank Experiments, TRANSACTIONS of The WESTJAPAN SOCIETY OF NAVAL ARCHITECTS, No.98, pp 151 - 160. (1999).

[4] Y.Nagashima, N.Taguchi, T.Ishimatsu and H.Inoue: Development of Compact Autonomous Underwater Vehicle Using VARIVEC Propeller, Proc. of JSME Robotics and Mechatronics Conference '01, pp 1A1 L1-1A1 - L2. (2001).

[5] T.Ura and S.Takagawa: Underwater Robots, Seizando Publishing Co. Tokyo

[6] K.Watanabe, T.Mishima, T.Nakano, M.Inagaki and Y.Iwasaki Development of "Varivec Propeller" Three Dimensional Propulsion Unit $1^{\text {st }}$. Report “, Review of Kawasaki Heavy Industries, Volume No.101, pp 23 30. (1988).

[7] K.Watanabe, Y.Iwasaki, Y.Tanaka, T.Idoko, M.Hino and M.Okada: Development of "Varivec Propeller" Three Dimensional Propulsion Unit $2^{\text {nd }}$. Report “, Review of Kawasaki Heavy Industries, Volume No.104, pp 30 - 38. (1989). 